\title{
Model Based Approach for Identification of Relevant Images from Ancient Paintings
}

\author{
G.G.Naidu ${ }^{\mathrm{a}}$, Y.Srinivas ${ }^{\mathrm{b} *}$ \\ ${ }^{a}$ Dept of Computer Engg, Govt Polytechnic, Anakapalli, Visakhapatnam - 531001, India \\ ${ }^{b}$ Dept of IT, GITAM University, Visakhapatnam -530045, India
}

Received: 12 January 2016; Accepted: 28 March 2017; Published: 08 September 2017

\begin{abstract}
In this paper an attempt is made to retrieve the relevant paintings based on the approach of the artist using Generalized Bivariate Laplacian Mixture Model (GBLMM). This article helps in understanding the outline of assorted artists and help as a means to categorize a scrupulous painting based on the style or the text ingrained within the images. To profile the artist style GBLMM is used. The projected model helps to discriminate the strokes of the artists and lend a hand in the classification of paintings. The proposed model is implemented using high resolution Chinese painting images.
\end{abstract}

Index Terms: GBLMM, classification, painting, retrieval, stroke.

(C) 2017 Published by MECS Publisher. Selection and/or peer review under responsibility of the Research Association of Modern Education and Computer Science.

\section{Introduction}

The area of image processing helps in better understanding the inherent details within the images. With the evolution of technologies, efforts are initiated to digitize the ancient arts and paintings [1]. Every artist holds his individual means of narration and depicts the same in the form of paintings. One can analyze the paintings that are digitized using the internet facility. Accordingly different algorithms have been developed to analyze the ancient digitized paintings [2]. Meaningful deductions can be made from the available literature, in better understanding the narrative styles of the artists. However, each painting has its own features, and each artist has his own way of drawing patterns, thereby the strokes applied among the artists differ. Several researchers have proposed methodologies to identify and classify the paintings based on features, digital signatures, frequency of application of a particular stroke etc. [3],[4],[5].

* Corresponding authors.

E-mail addresses: gagona.ggn@gmail.com, sriteja.y@gmail.com 
Among these models, models based on parametric and non-parametric are focused. However, most of the presented works are totally based on the classification, and identifying the relevancy based on particular metrics [3]. Moreover, while classifying the images based on features, the variation among the pixels is not taken into account. Very little importance is highlighted towards the loss of connectivity among the pixels. Therefore effective classification of the relevant images based on either artistic styles or based on specific artist cannot be categorized effectively. In this proposed article an attempt is made to bridge these gaps in this direction.

To every image, a Meta tag is associated and each the images along with the tags are modelled using GBLMM. The classification process is carried out using the maximum likelihood estimate. The rest of the paper is organized as follows. In section II of the paper, the related work is presented. The section III highlights the major challenges with respect to the retrieval of relevant images. Section IV of the paper presents the GBLMM model along with the methodology. Section $V$ highlights the feature extraction and the dataset considered together with experimentation is highlighted in section VI of the paper. The results derived together with evolution are highlighted in section VII and the final section VIII concludes the paper.

\section{Related Work}

Many authors have highlighted the methodologies for effective identification of the artistic images from the ancient paintings. C.C.Chen et al. (2002), have presented an idea of the ancient document together with the databases. Bouman, Liu (1991), have utilized the Markov model for segmenting the images. Choi and Baraniuk (1999), considered the hidden Markovian models for segmenting the images. The concept of gray scale document segmentation is utilized by Chaddha et al. (1994). The concept of thresh holding, VQ models, Bayes classification, segmentation of half toning are proposed by A.P.Dhawan et al. (1996), Sherbini (1995), Groes et al. (1995), Loum G et al. (1996), Mallet S.G (1989), F.Y.Shih (1996). In the article presented by Razvan George Condorovici et al (2013), the authors presented a model for automatic system that recognises Artistic genre identification. This is an integral part of the effective image processing systems aimed towards better understanding of artistic view. A relevance feedback mechanism was highlighted by Bogdan Boteanu et al (2014) in which the authors tried to retrieve the relevance images based on the query, it integrated the approaches of automation power of machines together support vector machine and relevance feedback mechanisms. Parag Dhonde et al (2015) have presented a model of image mining using the concepts of hierarchical K-Means algorithm and image vision tools to retrieve the images of interest. The concepts of parametric modelling were considered for classification of images and an elaborate study was presented by the authors Srinivas.Y (2007), Nagesh V (2010), Madhusudhan T (2013) and Anuradha P (2014).

\section{Major Challenges}

In spite of extensive works in this area, effective segmentation and classification is still a concern. This is due to fact that the features are extracted locally basing on which the segmentation is carried out, which results into core technical problems in case of similar images. The second challenge is with respect to the breaks in the images, in some of the images the variation among the inks cannot be identified exactly because of a low changes in the colour tones. Strokes of the artist also play vital role and very little attention is paid to identify the exactness of the strokes. To overcome this challenges, in this paper we have considered the GBLMM.

\section{Generalized Bivariate Laplacian Mixture Model}

The Probability Density Function (PDF) of GBLMM is given as

$$
f(x, y)=\frac{1}{\pi \sigma_{1} \sigma_{2} \sqrt{\left(1-\rho^{2}\right)}} \cdot k_{0} \cdot \sqrt{\left.\left(2\left(x^{2} / \sigma_{1}^{2}-2 p x y\right) /\left(\sigma_{1} \sigma_{2}\right)+\frac{y^{2}}{\sigma_{2}{ }^{2}}\right) /\left(1-\rho^{2}\right)\right)}
$$


Where

$$
k_{0} \text { is the Bessel function, } \rho \text { is scale parameter, } 0 \leq \rho \leq 1
$$

The main consideration of GBLMM is that its ability to identify the variation among the regions with in the images. The image is divided into parts using discrete wavelet Harr transformation and the different regions LL, LH, HL, and HH are identified. The LL band helps to identify the low variations inside the images, variations along the horizontal direction can be attributed using HL bands, LH bands are considered for changes along the vertical direction and $\mathrm{HH}$ are utilized for identifying the high intensity image regions.

Each image is considered and the histograms are extracted using which the various bands such as LL, LH, $\mathrm{HL}$ and $\mathrm{HH}$ are identified. Each of the pixels within these bands is identified and is modelled using the GBLMM and the relevant PDF are identified. In order to minimize the space complexity, each of these bands are further subdivided and the highest frequency values are considered from each of these such bands and these values are given as inputs to the PDF of GBLMM given in section 3 of the paper. This is called the training phase. In the testing phase, the relevant images are extracted by comparing the similarity among the PDFs for which KL-divergence is considered. The derived images are subjected to quality metrics for identification of the similarities.

\section{Feature Extraction}

To extract the features the images divided into sub bands containing LL, LH, HL, and HH features. Each of these sub bands are further sub divided and a unique feature vector is identified from each of these sub bands. These unique vectors are given as input for modelling the images using GBLMM proposed section 3 of the paper.

\section{Dataset Considered}

In ordered to proposed methodology we have considered the ancient paintings of the Chinese artists from the internet and in particular we have considered the paintings of Van Gogh, Zhang daqian given in the report of C.C.Cheng et al. (1996).

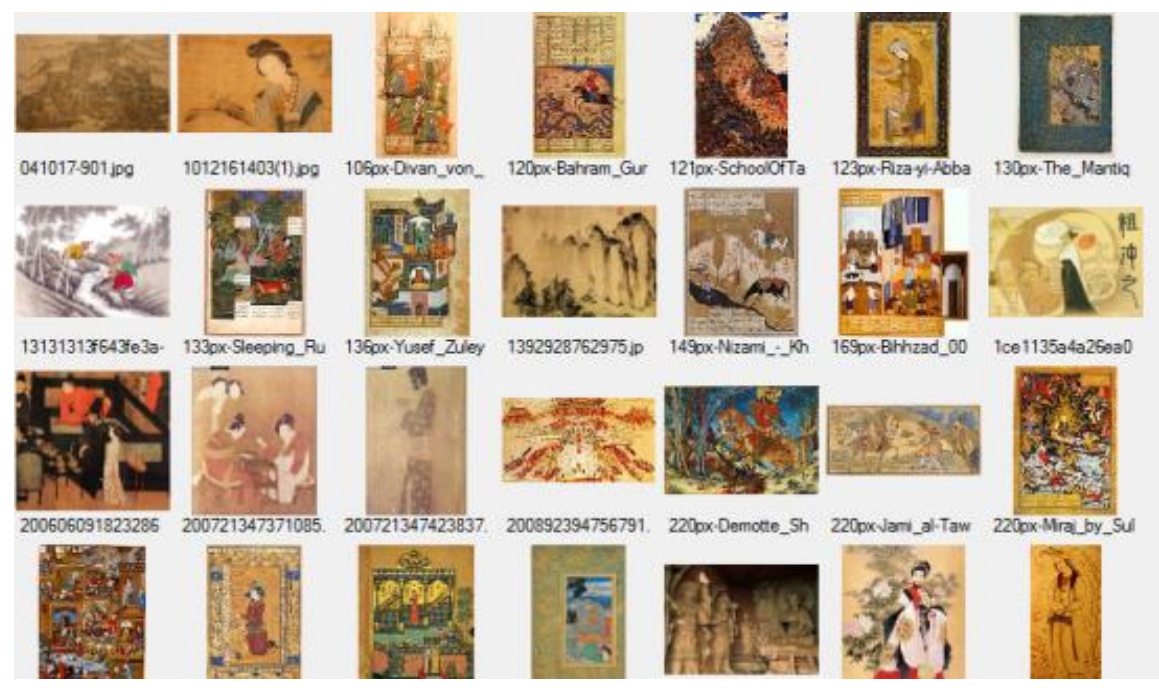

Fig.1. Dataset Considered 


\subsection{Meta Tags}

Every image considered is associated with a Meta tag. These Meta tags are given as another feature for the GBLMM as input. The various tags considered are presented in the following Table 1.

Table 1. Image with the Tags

\begin{tabular}{|c|c|}
\hline File Name & TAG \\
\hline 1ce1135a4a26ea08847bc18d7e4ef81e.jpg & ancient, Chinese, singing, song, red, horse cart, moon \\
\hline 4a1bc7599efb16e29d4e8cea94afbcaf.jpg & ancient, Chinese, king, palace \\
\hline Ancient-Chinese-gods-2.jpg & $\begin{array}{l}\text { ancient, Chinese, women, a tinge, vermeil, sweet hearted } \\
\text { ancient, Chinese, committee }\end{array}$ \\
\hline ancient-chinese-architecture-08.jpg & $\begin{array}{l}\text { ancient } \\
\text { ancient, Chinese, tang game }\end{array}$ \\
\hline ancient_chinese_women_e7df5562.jpg & ancient, Chinese, xianterra, cota, warriors \\
\hline Ancient-Chinese-Social-Ranks.jpg & $\begin{array}{l}\text { song,yingxing,chinese,scientist,encyclopedist and ,tiangong } \\
\text { kaiwu,an encyclopedia }\end{array}$ \\
\hline doctor.jpg & mohism,ancient,chinese,philosophy \\
\hline committee.jpg & ancient, Chinese, architecture, temple \\
\hline group3.jpg & ancient, Chinese, doctor \\
\hline images.jpg & ancient, Chinese, soldier \\
\hline making_of_jonathan_spence.jpg & ancient, Chinese, priest, education, scholarship \\
\hline mohism_ancient_chinese_philosophy.jpg & Ancient, Chinese, god \\
\hline song_yingxing_a_chinese_scientist.jpg & Ancient, Chinese, Social, Ranks \\
\hline xianterracotawarriors.jpg & ancient, Chinese, jonathan,spence,scholar \\
\hline 123px-Riza- Abbasi_008.jpg & $\begin{array}{l}\text { Saki, Reza Abbasi, Moraqqa e, Golshan, Golestan Palace, ancient, } \\
\text { Chinese }\end{array}$ \\
\hline 220px-Saki_-_Reza_AbbasiPalace.jpg & Riza, yi, Abbasi, ancient, Chinese \\
\hline 220px-Mir_Sayyid_Ali_2.jpg & Behzad, advice, ascetic, ancient, Chinese \\
\hline 300px-Behzad_advice_ascetic.jpg & ancient, Chinese, Mir Sayyid Ali story \\
\hline 220px-Jami_al-Tawarikh_Miraj.jpg & ancient, Chinese, Jami al Tawarikh Miraj \\
\hline
\end{tabular}




\section{Results}

In order to present the methodology, we have considered the dataset as discussed in section 6, the dataset consists of the paintings of ancient Chinese artists. Along with these paintings a narration about the paintings or the name of the artist is also included. In our methodology we have considered these descriptions as the tags and retrieval is carried out using these tags as a feature. Each image with the tag as index is given as input to the model and the corresponding Probability Density Functions (PDFs) generated against each of these images are noted in a matrix. In general there is a possibility of repetition of tags and therefore the retrieval efficiency degrades in most of the cases. In order to overcome this challenge, in the present model we have considered more than one tag as the index. The images that are retrieved against each of these tags is presented in the following Fig 2.

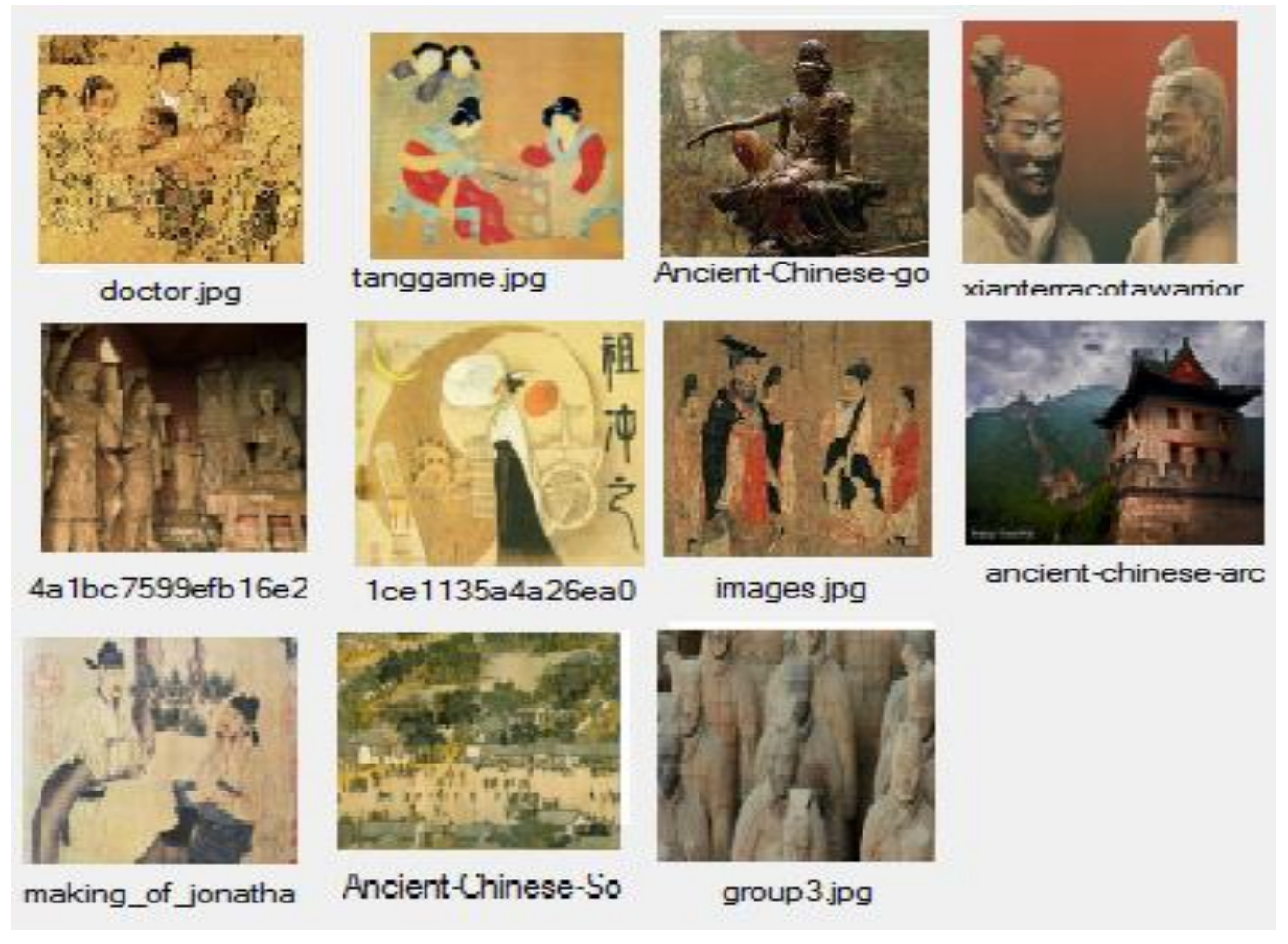

Fig.2. Retrieved images, based on Tags

The above methodology is tested against quality metrics such as Mean Squared Error (MSE), Peak Signal to Noise Ratio (PSNR) The formulas for calculation of each these metrics is presented in the paper authored by Srinivas Y et al (2010), Nagesh V et al (2011), Madhusudhan et al (2013). Using the above heuristics, the results derived are presented in Table 2.

From the above table it can be clearly seen that the MSE of the retrieved image against the query image is nearing to zero. This implies that the retrieved image is very much nearer to the query image. The PSNR values highlight the same. From the values presented in the Table 2, it can be seen that the developed model helps in better retrieval of the images. 
Table 2. Performance Evaluation

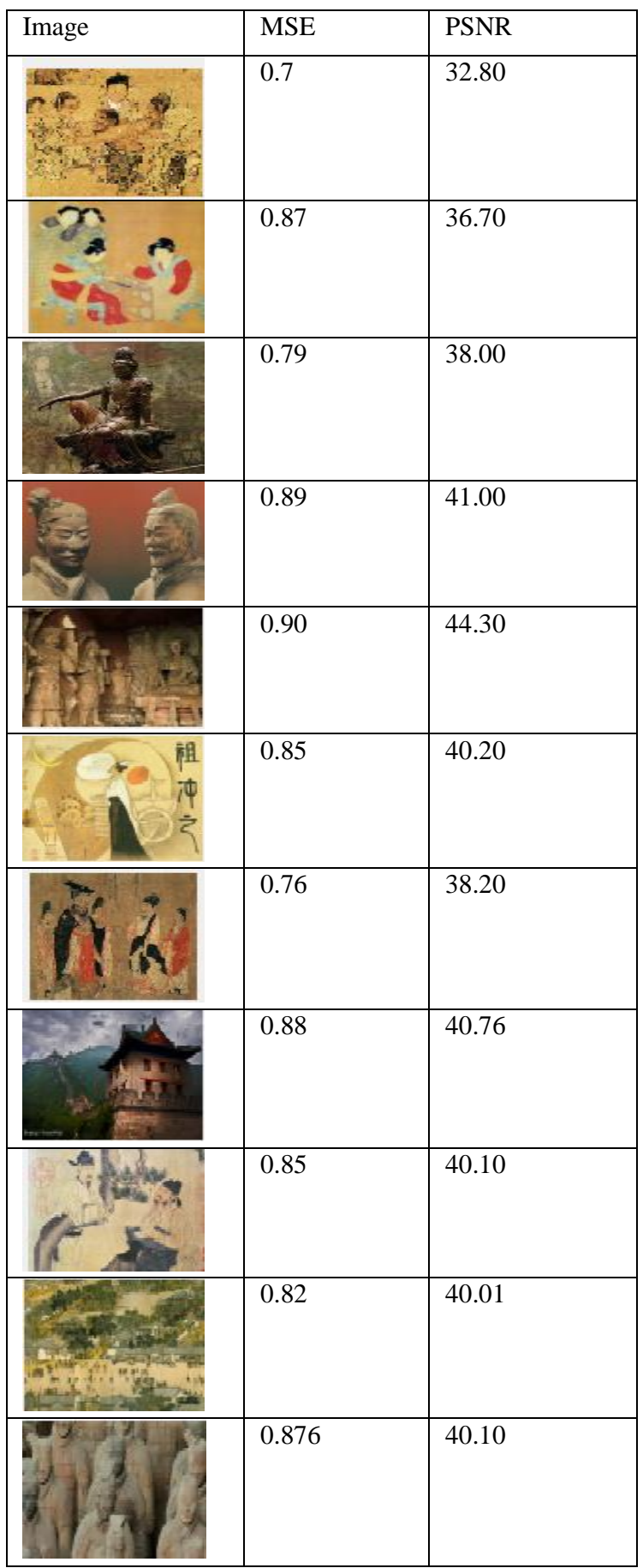




\section{Conclusions}

This paper highlights the methodology for the retrieval of ancient images based on the features which we have considered to be the tags. The proposed methodology helps for the better retrieval against the existing methods. This methodology is very much suited for the archaeologists for identifying the ancient paintings of a particular artist.

\section{References}

[1] C.-C. Chen, A. Del Bimbo, G. Amato, N. Boujemaa, P. outhemy, J.Kittler, I. Pitas, A. Smeulders, K. Alexander, K. Kiernan, C.-S. Li, H.Wactlar, and J. Z. Wang, "Report of the DELOS-NSF Working Group on Digital Imagery for Significant Cultural and Historical Materials," DELOS-NSF Rep., Dec. 2002

[2] C. Bouman and B. Liu, "Multiple resolution segmentation of textured images," IEEE Trans. Pattern Anal. Machine Intell., vol. 13, no. 2, pp.99-113, 1991

[3] S. Ravela and R. Manmatha, "Image retrieval by appearance," in Proc.SIGIR, Philadelphia, PA, July 1997, pp. 278-285.

[4] A. P. Dhawan, Y. Chitre, C. Kaiser-Bonasso, and M. oskowitz, "Analysis of mammographic microcalcifications using gray-level image structure features," IEEE Trans. Med. Imag., vol. 15, pp. 246259, June 1996

[5] N. Chaddha, R. Sharma, A. Agrawal, and A. Gupta, "Text segmentation in mixed-mode images," in Proc. Asilomar Conf. Signals, Systems, Computers, vol. 2, Nov. 1994, pp. 1356-1361

[6] R.S. Arora, A. Elgammal, Towards automated classification of fine-art painting style: a comparative study, in: ICPR, 2012, pp. 3541-3544.

[7] Y. Bengio, P. Lamblin, D. Popovici, H. Larochelle, Greedy layer-wise training of deep networks, in: NIPS, 2006, pp. 153-160.

[8] M. Bressan, C. Cifarelli, F. Perronnin, An analysis of the relationship between painters based on their work, in: ICIP, 2008, pp. 113-116.

[9] C.C. Chang, C.J. Lin, LIBSVM: a library for support vector machines, ACM Trans. Intell. Syst. Technol. 2 (2011) 27:1-27:27.

[10] R. Condorovici, C. Florea, R. Vranceanu, C. Vertan, Perceptually-inspired artistic genre identification system in digitized painting collections, Image Analysis, LNCS, vol. 7944, Springer, 2013. 687-696.

[11] T. Fawcett, An introduction to ROC analysis, Pattern Recogn. Lett. 27 (2006) 861-874.

[12] V. Ferrari, L. Fevrier, F. Jurie, C. Schmid, Groups of adjacent contour segments for object detection, IEEE TPAMI 30 (2008) 36-51.

[13] D.J. Graham, J.M. Hughes, H. Leder, D.N. Rockmore, Statistics, vision, and the analysis of artistic style, Wiley Interdisciplinary Rev. Comput. Stat. 4 (2012) 115-123.

[14] J. Li and J.Z. Wang, "Studying digital imagery of ancient paintings by mixtures of stochastic models," IEEE Trans. Image Processing, vol. 13, no. 3, pp. 340-353, Mar.2004.

[15] E.Hendriks and M. Geldof, "Van Gogh's Antwerp and Paris picture supports (1885-1888): Reconstructing choices," Art Matters: Netherlands Technical Studies in Art History, vol. 2, pp. 39-75, 2005.

[16] E. Hendriks and L. van Tilborgh, "New views on Van Gogh's development in Antwerp and Paris: An integrated art historical and technical study of his paintings in the Van Gogh Museum," Ph.D. dissertation, Faculty of Humanities, Univ. Amsterdam, vol. 1, p. 132, and vol. 2, p. 62, cat. 72, Nov.2006.

[17] C.R. Johnson, Jr., Ed., Proc. 1st Int. Workshop Image Processing for Artist Identification. Amsterdam, The Netherlands, May 2007 [Online]. Available: http://digitalpaintinganalysis.org/literature/ProceedingsIA4AI-1.pdf 
[18] Thai V. Hoang, S. Tabbone(2010),“Text Extraction From Graphical Document Images Using Sparse Representation "in Proc. Das, pp 143-150.

[19] Y. Zhan, W. Wang, W. Gao (2006), “A Robust Split-And-Merge Text Segmentation Approach For Images", International Conference On at tern Recognition,06(2): pp 1002-1005.

[20] M.M. Van Dantzig, Vincent A New Method of Identifying the Artist and His Work and of Unmasking the Forger and His Products. Amsterdam, The Netherlands: Keesing, 1952, pp. 24-25.

[21] Zhang D, Lu G. A comparative study of curvature scale space and Fourier descriptors for shape-based image retrieval. J Visual Common Image Representation 2003; 14:41-60.

[22] F.Cheng, H.Zhang, M.Sun, D.Yuan, "Cross-trees, edges and super-pixel priors-based cost aggregation for stereo matching", in Pattern Recognition 48 (2015) 2269-2278.

[23] K. Andreas, M. Sormann, K. Karner, "Segment-based stereo matching using belief propagation and a selfadapting dissimilarity measure", in: International Conference on Pattern Recognition, vol. 3, 2006, pp. $15-18$.

[24] F. Cheng, H.Zhang, D.Yuan, M.Sun, "Stereo matching by using the global edge constraint", in Neurocomputing (2013).

[25] X.Wang, H.Wang, Y.Su, "Accurate belief propogation with parametric and non-parametric measures for stereo-matching", in Optik 126(2015) 545-550.

[26] F.Da, F.He, Z.Chen, "Stereo Matching based on dissimilar intensity support and belief propogation", in J Math Imaging Vis (2013) 47:27-34.

[27] F. Tombari, S. Mattoccia, L. Di Stefano, E. Addimanda, "Classification and evaluation of cost aggregation methods for stereo correspondence", in Proceedings of the IEEE Conference on Computer Vision and Pattern Recognition, 2008, pp. 1-8.

[28] H.Han, X.Han, F.Yang, "An improved gradient-based dense stereo correspondence algorithm using guided filter",in Optik125 (2014),pp.115- 120.

[29] F.Tombari, S.Mattoccia, L.Stefano Di, "Segmentation-Based Adaptive Support for Accurate Stereo Correspondence", in PSIVT 2007, LNCS 4872, pp. 427-438, 2007.

[30] O. Veksler, "Stereo correspondence by dynamic programming on a tree", in: IEEE International Conference on Computer Vision and Pattern Recognition, 2005, pp. 384-390.

[31] R.Elias, "Sparse view Stereo Matching”, in Pattern Recognition Letters 28 (2007) 1667-1678.

[32] S.Yoon, S.K.Park, Y.K.Kwak, "Fast correlation-based stereo matching with the reduction of systematic errors", Pattern Recognition Letters 26(2005), pp.2221-2231.

[33] K.Yoon, I.So.Kweon, "Adaptive Support-weight approach for correspondence search", IEEE Transactions on Pattern Analysis and Machine Intelligence(2006),vol.28,no.4.

[34] P.H.S.Torra, A.Criminisi, "Dense stereo using pivoted dynamic programming", Image and Vision Computing 22(2004), pp.795-806.

[35] M.Bleyer, M.Gelautz, “ A layered stereo matching algorithm using image segmentation and global visibility constraints", ISPRS Journal of Photogrammetry \& Remote Sensing 59(2005),pp.128-150

[36] O.Veksler, "Extracting dense features for visual correspondence with graph cuts", Proceedings of the IEEE Computer Society Conference on Computer vision and Pattern Recognition (2003).

[37] M.Gong, Y.H.Yang, "Fast stereo matching using reliability based dynamic programming and consistency constraints", Proceedings of ninth IEEE International Conference on computer vision (2003).

[38] C. L. Zitnick and T. Kanade, "A Cooperative Algorithm for Stereo Matching and Occlusion Detection," IEEE Trans. Pattern Analysis and Machine Intelligence, vol. 22, no.7, 2000.

[39] J. Sun, H. Y. Shum and N. N. Zheng, "Stereo Matching Using Belief Propagation,"Proc. European Conf. Computer Vision (2002), pp. 510-524.

[40] V. Komolgorov and R. Zabih, "Computing Visual Correspondence with Occlusions using Graph Cuts", Proc. Int' 1 Conf. Computer Vision (2002).

[41] Y. Boykov, O. Veksler and R. Zabih, "Fast Approximate Energy Minimization via GraphCuts", IEEE Trans. Pattern Analysis and Machine Intelligence (2001), vol. 23, no. 11, pp. 1222-1239. 
[42] S. Birchfield and C. Tomasi, "Depth discontinuities by pixel-to-pixel stereo", In ICCV (1998), pages 1073-1080.

[43] Z. Yongqin, C.Hui, W. Ling, X.Yongjun, H.Haibo, "Color Image Segmentation Using Level Set Method With Initialization Mask in Multiple Color Spaces", I.J. Engineering and Manufacturing, 2011, 4, 70-76.

[44] Ramandeep Kaur, Kamaljit Kaur, "Study of Image Enhancement Techniques in Image Processing: A Review”, I.J. Engineering and Manufacturing, 2016, 6, 38-50.

\section{Author's Profiles}

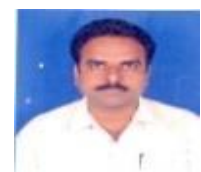

G.G.Naidu has completed his M.Tech Degree in Computer Science and Engineering, JNT University, Kakinada. His research area includes Image Retrieval, Data Mining. Currently he is working as Head of Computer Engineering section, Govt Polytechnic, Anakapalli, Visakhapatnam, India.

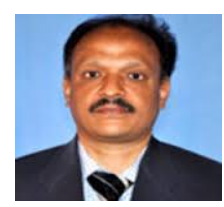

Y. Srinivas is presently working as a Professor and Head of Department of Information Technology, GITAM University, Visakhapatnam, India. His research area includes Image Processing, Data Mining, Software Engineering and Digital forensics.

How to cite this paper: G.G.Naidu, Y.Srinivas,"Model Based Approach for Identification of Relevant Images from Ancient Paintings", International Journal of Engineering and Manufacturing(IJEM), Vol.7, No.5, pp.3947, 2017.DOI: 10.5815/ijem.2017.05.04 